\title{
Assessment of Computed Tomography Images for the Diagnostic Yield of Endobronchial Ultrasonography with a Guide-Sheath for Ground-Glass Opacity Lesions
}

\author{
Mariko Okamoto $^{1}$, Noriaki Kurimoto ${ }^{2}$, Kei Morikawa ${ }^{1}$, Shin Matsuoka ${ }^{3}$, \\ Takeo Inoue $^{1}$, Masamichi Mineshita ${ }^{1}$, and Teruomi Miyazawa ${ }^{1}$
}

(Received for Publication: August 19, 2015)

\begin{abstract}
Background: Biopsy of ground-glass opacity (GGO) lesions performed under endobronchial ultrasonography with a guide sheath (EBUS-GS) has produced relatively low diagnostic yields.

Objectives: To clarify whether CT findings can contribute to the return of an EBUS-GS-guided diagnosis of GGO lesions.

Methods: We retrospectively evaluated 58 patients with GGO lesions. Forty-four of 58 lesions were diagnosed by means of EBUS-GS-guided biopsy, and the remaining 14 undiagnosed by EBUS-GS-guided biopsy were diagnosed by CT-guided biopsy or surgery. We reviewed CT images obtained prior to EBUS-GS-guided biopsy to measure the maximum diameter of the GGO lesion, the maximum diameter of the solid component, the distance from the bifurcation of the lobe bronchus to the lesion, and the presence or absence of a "bronchus sign."

Results: The diagnostic yield for lesions with a maximum diameter of the solid component $>5 \mathrm{~mm}(38 / 46$, $82.6 \%$ ) was significantly greater than that for the lesions with a maximum diameter of the solid component $\leq 5$ $\mathrm{mm}(6 / 12,50 \%)(p=0.024)$. There was no significant difference in the diagnostic yield based on the maximum GGO diameter, the distance from the bifurcation of the lobe bronchus to the lesion, or the presence of a bronchus sign.
\end{abstract}

Conclusions: Our data suggest that EBUS-GS-guided biopsy can yield a diagnosis when the maximum diameter of the solid part of the malignant GGO lesion depicted on the CT image is $>5 \mathrm{~mm}$.

Key words

bronchoscopy, endobronchial ultrasonography, guide sheath, ground-glass opacity, lung cancer diagnosis

\section{Introduction}

The number of early-stage lung cancers detected by computed tomography (CT) screening is high, and such screening is reported to decrease lung cancer mortality rates ${ }^{1}$. A high percentage of well-differentiated adenocarcinomas manifest as ground-glass opacity (GGO) lesions, and this type of cancer is said to have a good prognosis. For a preoperative diagnosis of peripheral pulmonary lesions (PPLs), the Ameri- can College of Chest Physicians (ACCP) guidelines recommend CT-guided biopsy or bronchoscopic biopsy guided by endobronchial ultrasound (EBUS) using a high-frequency radial probe ${ }^{2}$.

In 2004, we reported the usefulness of endobronchial ultrasonography with a guide sheath (EBUS-GS) for collecting diagnostic samples from PPLs, including those too small to be visualized under fluoroscopy ${ }^{3)}$. We obtained favorable results, obtaining a diagnostic yield of $70 \%$ with EBUS-GS gui-

1 Division of Respiratory Medicine, Department of Internal Medicine

2 Division of Chest Surgery, Department of Surgery

3 Department of Radiology, St. Marianna University School of Medicine 
ded transbronchial biopsy for pulmonary lesions $\leq 10$ $\mathrm{mm}$ in diameter.

There have since been several reports on the use of EBUS-GS for the diagnosis of PPLs but few reports on the use of EBUS-GS for the diagnosis of pulmonary GGO lesions. Reports thus far have indicated that the visibility of GGO lesions during EBUS-GS contributes significantly to the ability of EBUS-GS-guided biopsy to return a diagnosis, with Ikezawa et al. ${ }^{4)}$ reporting a diagnostic yield of $57 \%$ for pulmonary GGO lesions and Izumo et al. ${ }^{5)}$ reporting a diagnostic yield of $65 \%$.

Prognosis is another important issue. The diameter of the solid part in a GGO lesion is reported to be an important prognostic factor in the surgical treatment of such lesions. Naturally, diagnosing nodules with a solid part at an early stage facilitates early treatment, and if we can elucidate specific CT findings that contribute to a diagnosis achieved by means of EBUS-GS-guided biopsy, we may be able to perform EBUS-GS proactively to obtain a diagnosis before a decision is made to perform surgery.

We conducted a retrospective study of patients with pulmonary GGO lesions evaluated by means of EBUS-GS-guided biopsy to identify particular CT findings that contribute to diagnosis.

\section{Methods}

\section{Patients}

This study was performed at St. Marianna University School of Medicine Hospital, with ethics approval from the Human Research and Ethics Committee (approval reference no. 2858). Between January 2010 and December 2013, consecutive 71 patients with pulmonary GGO lesions detected on chest CT underwent EBUS-GS at our hospital. A diagnosis was achieved by EBUS-GS-guided biopsy in 44 of these patients, but the diagnosis remained uncertain in 27 patients. Our study group comprised 58 patients, 44 with a diagnosis obtained by EBUS-GSguided biopsy and 14 of the 27 patients without a diagnosis before CT-guided biopsy or surgery was performed (Fig. 1). The remaining 13 patients were followed up without CT-guided biopsy or surgery and were not included in our study.

For the EBUS-GS-guided biopsy specimens of these remaining 13 patients that were judged to have inflammatory change or no malignancy on pathological examination, follow-up CT imaging was performed after 3 to 6 months. One lesion that spontaneously disappeared was designated as benign.

\section{Definition of GGO Lesion}

GGO is generally used to describe focal nodular areas of increased lung attenuation, including both well and poorly defined lesions, through which normal parenchymal structures appear without obscuring pulmonary vessels. Localized GGO is normally referred to as a ground-glass nodule (GGN). For this study, GGO lesions included lesions with the appearance of a pure GGN and those that appeared to have a solid part (part-solid GGN). In contrast to GGNs, part-solid GGNs have both ground glass and solid components, the latter obscuring the underlying lung architecture $^{6}$. Pure GGN is classified as having a solid-part diameter of 0-mm.

\section{CT and Measurement of GGO}

All patients underwent chest CT prior to bronchoscopic examination for evaluation of morphologic

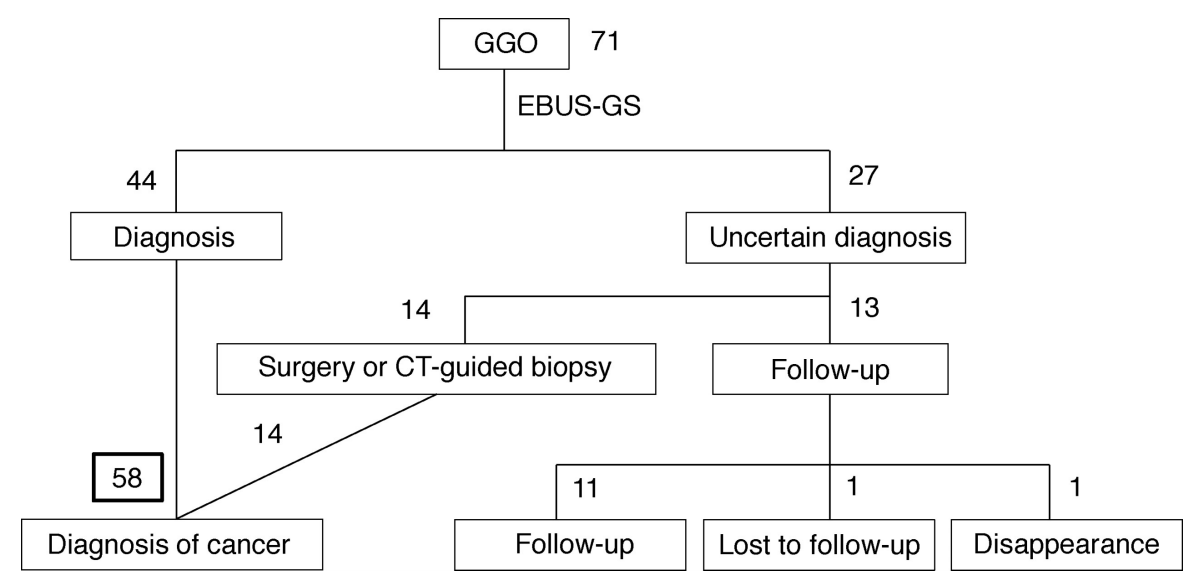

Figure 1. Study diagram. 
appearances. The CT examination was performed with a 16- or 64-row multi-detector CT scanner (Aquilion 16 or Aquilion 64, Toshiba Medical Systems, Tochigi, Japan). For each patient, CT was performed in the supine position at full inspiration (0.5$\mathrm{mm}$ collimation, 0.5 -s gantry rotation time). The tube voltage was fixed at $120 \mathrm{kVp}$, and the tube current was adjusted by automatic exposure control. CT images were contiguously reconstructed with a 1-mm slice thickness and an FC52 kernel. The lung window was adapted to a width of 1500 Hounsfield units (HU) and a window level of $-600 \mathrm{HU}$. No intravenous contrast material was used.

On the selected CT images, all slices containing the focal lesion were examined by a pulmonologist with 6 years experience. The maximum diameter of both the GGO and the solid part were measured (Fig. 2). We evaluated the distance from the bifurcation of the lobe bronchus to the lesion on multiplanar reconstruction images (Ziostation2, Ziosoft, Inc., Tokyo, Japan) and the presence or absence of a "bronchus sign" (the finding on cross-sectional view of a bronchus leading directly to or contained within the nodule). High-resolution CT images were reviewed by a pulmonologist with 6 years of experience, a thoracic surgeon with 30 years of experience, and a board-certified radiologist to assess the bronchus sign ${ }^{7}$.

\section{Bronchoscopy and EBUS-GS}

Bronchoscopic examination was performed with a bronchoscope having a 4-mm external diameter and 2-mm working channel (BF-P260F, Olympus, Tokyo, Japan). A $20-\mathrm{MHz}$ radial-type ultrasound probe (UM-S20-17S, Olympus) with a guide sheath (Guide Sheath Kit, K-201, Olympus) was inserted through the working channel of the bronchoscope. The bronchoscopist and chest surgeon reviewed the CT images to determine in which bronchus to insert the probe.

The bronchoscopy procedure was performed under sedation with local anesthesia, and the bronchoscope was inserted through the patient's mouth or nose. The bronchoscopist first explored whether additional lesions were present in the bronchus within the field of view. The tip of the bronchoscope was then inserted into the target bronchus, and the ultrasound probe, which was covered by the guide sheath, was inserted into the bronchus. Scanning was performed under X-ray fluoroscopic guidance, and EBUS images were obtained. The positional relations between the probe and the focal lesion were assessed as fol-

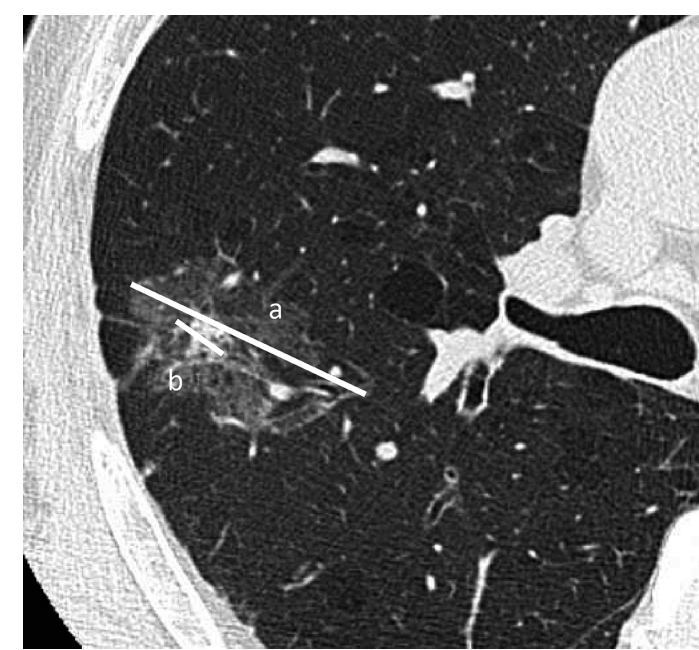

Figure 2. Measurement of GGO.

The maximum GGO diameter (a) and maximum solid-part diameter (b) on CT images were measured.

lows: whether the probe was located within the lesion (noted as "within"), whether the probe was in contact with the lesion (noted as "adjacent to"), or whether the probe was not in contact with the lesion (noted as "invisible"), making it impossible to image the focal lesion ${ }^{3)}$

In addition, we applied the classification system used by Kurimoto et al. for EBUS features of PPLs ${ }^{8}$. This system classifies PPLs according to the heterogeneity of the internal echo, the vascular and bronchial patency, and the morphology of the hyperechoic areas. We applied the following classifications: Type I, representing a homogenous echo pattern; Type II, representing a pattern of hyperechoic dots and linear arcs; and Type III, representing a heterogeneous echo pattern. Kurimoto et al. reported that well-differentiated adenocarcinomas commonly fall into Type II. Thus, Type II was subdivided into Type IIa (hyperechoic dots and linear arcs without vessels) and Type IIb (hyperechoic dots and linear arcs with patent vessels) (Fig. 3).

After confirming the location of the target lesion with EBUS-GS, we withdrew the probe, leaving the guide sheath in place at the lesion site. Brushing cytology and forceps biopsy were performed 3-5 times to collect samples before a bronchial wash was performed (Fig. 4). After removal of the guide sheath from the bronchoscope, we flushed the guide sheath lumen with approximately $2 \mathrm{~mL}$ of physiological saline solution and mixed this with the fluid from the 

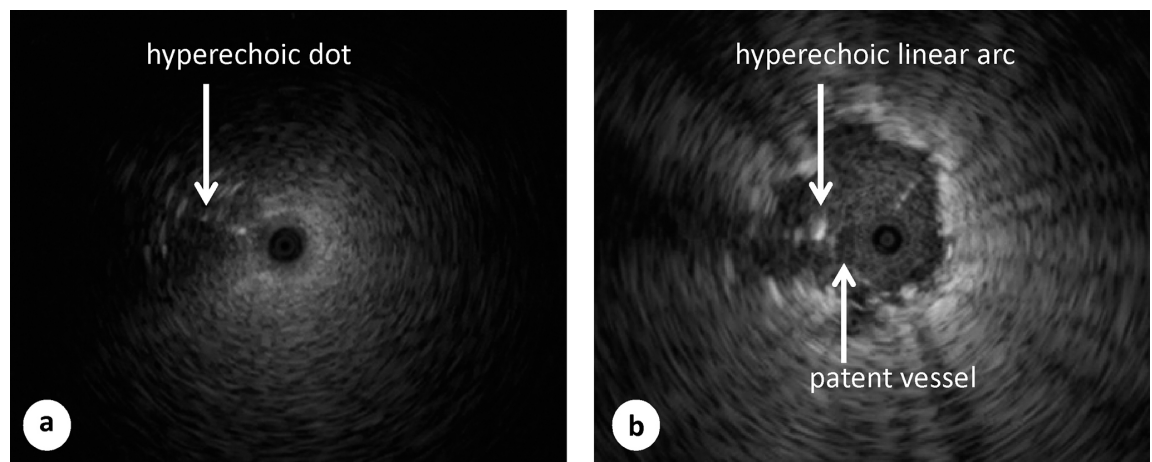

Figure 3. Type II EBUS image.

a: A representative Type IIa lesion showed hyperechoic dots and linear arcs without vessels.

b: A representative Type IIb lesion showed hyperechoic dots and linear arcs with patent vessels.

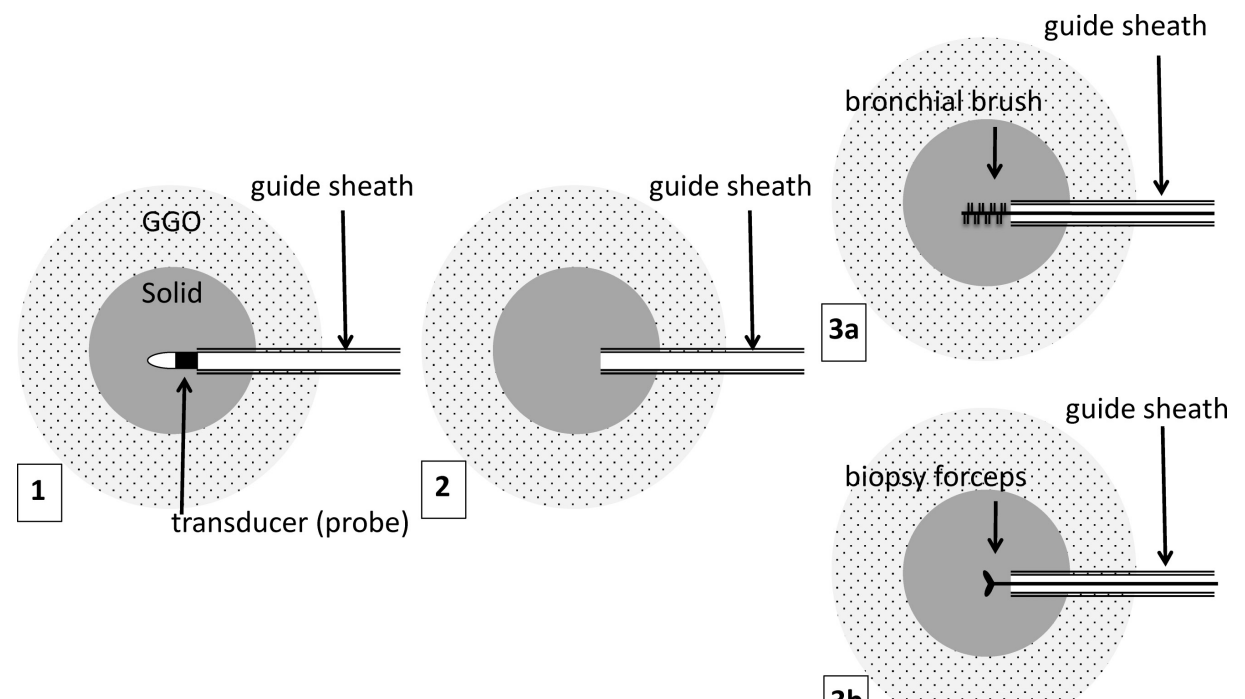

$3 \mathbf{b}$

Figure 4. EBUS-GS Procedure.

1: The tip of the bronchoscope is inserted into the target bronchus, and the ultrasonic probe, which is covered by the guide sheath, is inserted into the bronchus to locate the lesion.

2: When the lesion is found, the probe is removed with the guide sheath left in situ in the lesion.

3: Brushing (a) and forceps biopsy (b) are performed 3-5 times to collect samples.

bronchial wash. If the target was invisible by EBUS, we did not perform a forceps biopsy.

An experienced pathologist examined the brushing samples and bronchial wash solution for cytologic diagnosis, and an experienced pathologist examined biopsy samples for histologic diagnosis. A lesion was considered to be malignant if the cytology specimen was positive for cancerous cells or if the biopsy specimen was found to be cancerous. Diagnostic yield was defined as the total number of patients with a diagnosis by EBUS-GS divided by the total number of diagnoses by either EBUS-GS (including 
invisible cases in which we did not perform forceps biopsy), CT-guided biopsy, or surgery. Patients for whom the bronchoscopy specimens were not judged to be malignant underwent CT-guided biopsy or surgery, or they were simply followed up, and any specimen obtained during CT-guided biopsy or surgery that was cytologically or histologically positive for cancerous cells was considered malignant.

For the 58 patients with a diagnosis obtained by EBUS-GS-guided biopsy, surgery, or CT-guided biopsy, CT findings were examined to determine whether the maximum GGO diameter, the maximum solid-part diameter, the distance from the bifurcation of the lobe bronchus to the lesion, and/or the presence of a bronchus sign, predicted a EBUS-GS-guided biopsy diagnosis.

\section{Statistical Analysis}

Descriptive statistics are presented as frequency, percentage, mean \pm standard deviation (SD), or median values. Polynomial logistic regression analysis was used to determine whether the maximum GGO diameter ( $\leq 20 \mathrm{~mm}$ vs. $>20 \mathrm{~mm}$ ), the maximum solidpart diameter ( $\leq 5 \mathrm{~mm}$ vs. $>5 \mathrm{~mm}$ ), the distance from the bifurcation of the lobe bronchus to the lesion, and/or the presence of a bronchus sign influenced the diagnostic yield. Fisher's exact test (2-sided) was used to examine whether the location of the EBUSGS probe influenced the EBUS-GS-guided diagnosis and whether the diameter of the solid part ( $\leq 5 \mathrm{~mm}$ vs. $>5 \mathrm{~mm}$ ) and the EBUS classification (Type IIa and Type IIb) influenced the diagnosis. SPSS version 19.0.0 (IBM Japan, Ltd., Tokyo, Japan) was used for all analyses and $p$ values of $<0.05$ were considered significant. Ryan's method in R version 2.4.1 (The R Project for Statistical Computing, Vienna, Austria) was used to examine the relation between EBUS classification and the diagnostic yield.

\section{Results}

The total study group comprised 28 men and 30 women, aged $68.7 \pm 9.0$ years. Lesion diameters were
$26.7 \mathrm{~mm} \pm 9.9 \mathrm{~mm}$, and all lesions were diagnosed as adenocarcinoma. Of the 58 lesions, 10 were found to be pure GGN and 48 were found to be part-solid lesions. The diagnostic yield of EBUS-GS among the 58 patients was $75.9 \%(44 / 58)$, including pure GGNs $(5 / 10,50 \%)$ and part-solid lesions $(39 / 48,81.3 \%)$. Complications such as pneumothorax or hemorrhage were not observed after the EBUS-GS procedure.

Twenty-five (56.8\%) of the 44 EBUS-GS-guided diagnoses were derived from both cytologic and histologic examinations, and the other 19 were derived from either cytologic examination $(\mathrm{n}=3,6.8 \%)$ or histologic examination $(\mathrm{n}=16,36.4 \%)$.

The maximum GGO diameters and the maximum solid-part diameters of the 58 lesions, classified by size, are shown in Table 1.

Polynomial logistic regression analysis revealed a significant difference between the diagnostic yield of lesions with a maximum solid-part diameter $>5$ $\mathrm{mm}(38 / 46,82.6 \%)$ and that of the lesions with a maximum solid-part diameter $\leq 5 \mathrm{~mm}(6 / 12,50 \%)(p$ $=0.024$ ) (Table 2). Of the 12 lesions with a solid part $\leq 5 \mathrm{~mm}, 2(2 / 12,16.7 \%)$ were invisible and $10(10 / 12$, $83.3 \%$ ) were visible on EBUS. Of the 46 lesions with a solid part $>5 \mathrm{~mm}, 3(3 / 46,6.5 \%)$ were invisible and 43 (43/46, 93.5\%) were visible on EBUS. The 5 cases of invisible lesions are listed in Table 3. Two of the 5 cases were of pure GGN and had a negative bronchus sign. We did not perform forceps biopsy in any of the 5 invisible cases.

The EBUS lesions sorted according to diagnostic yield per EBUS classification are shown in Table 4. Lesions are classified on this table by the size of the solid portion, either $\leq 5 \mathrm{~mm}$ or $>5 \mathrm{~mm}$. The proportion of Type IIa lesions in the patients with a solid component $\leq 5 \mathrm{~mm}(10 / 10,100 \%)$ and the proportion of Type IIb lesions in the patients with a solid component $>5 \mathrm{~mm}(29 / 43,67.4 \%)$ were significantly high $(p<0.01)$. The diagnostic yield for Type IIa lesions with a solid component $\leq 5 \mathrm{~mm}$ was $60 \%$ (6/10), that for Type IIa lesions with a solid component $>5 \mathrm{~mm}$ was $92.9 \%$ (13/14), and that for Type IIb with a solid

Table 1. CT Features of the 58 Lesions

\begin{tabular}{lccc}
\hline GGO diameter & \multicolumn{3}{c}{ Solid-part diameter $(\mathrm{mm})$} \\
& 0 & $>0$ to $\leq 5$ & $>5$ \\
\hline$\leq 20 \mathrm{~mm}(\mathrm{n}=16)$ & 3 & 1 & 12 \\
$>20 \mathrm{~mm}(\mathrm{n}=42)$ & 7 & 1 & 34 \\
Total $\quad(\mathrm{n}=58)$ & 10 & 2 & 46 \\
\hline CT $=$ computed tomography. &
\end{tabular}


Table 2. Logistic Regression Analysis of CT Features Influencing Diagnostic Yield

\begin{tabular}{|c|c|c|c|}
\hline & No. / Total no. [\%] & Odds ratio $[95 \% \mathrm{Cl}]$ & $p$ Value \\
\hline Diagnosis & $44 / 58$ [75.9] & & \\
\hline \multicolumn{4}{|l|}{ GGO diameter } \\
\hline$\leq 20 \mathrm{~mm}$ & $9 / 16[56.3]$ & \multirow{2}{*}{3.961 [0.798-19.645] } & \multirow{2}{*}{0.092} \\
\hline$>20 \mathrm{~mm}$ & $35 / 42[83.3]$ & & \\
\hline \multicolumn{4}{|l|}{ Solid-part diameter } \\
\hline$\leq 5 \mathrm{~mm}$ & $6 / 12[50.0]$ & \multirow{2}{*}{$5.615[1.255-25.124]$} & \multirow{2}{*}{0.024} \\
\hline$>5 \mathrm{~mm}$ & $38 / 46$ [82.6] & & \\
\hline \multicolumn{4}{|l|}{ Bronchus sign } \\
\hline+ & $36 / 45$ [80.0\%] & \multirow{2}{*}{$1.208[0.222-6.574]$} & \multirow{2}{*}{0.827} \\
\hline- & $8 / 13[61.5 \%]$ & & \\
\hline \multicolumn{4}{|l|}{ Distance from } \\
\hline bifurcation of lobe & & & \\
\hline $\begin{array}{l}\text { bronchus to lesion } \\
(\mathrm{mm})\end{array}$ & & $0.986[0.956-1.016]$ & 0.359 \\
\hline
\end{tabular}

Table 3. Invisible Cases on EBUS-GS

\begin{tabular}{ccccc}
\hline Case & $\begin{array}{c}\text { GGO } \\
\text { diameter } \\
(\mathrm{mm})\end{array}$ & $\begin{array}{c}\text { Solid-part } \\
\text { diameter } \\
(\mathrm{mm})\end{array}$ & $\begin{array}{c}\text { Bronchus } \\
\text { sign }\end{array}$ & $\begin{array}{c}\text { Distance } \\
\text { from } \\
\text { bifurcation } \\
\text { of lobe } \\
\text { bronchus to } \\
\text { lesion (mm) }\end{array}$ \\
\hline 1 & 13 & 0 & - & 82 \\
2 & 14 & 0 & - & 62.7 \\
3 & 19 & 6 & + & 97.2 \\
4 & 19 & 12 & - & 97.9 \\
5 & 26 & 16 & + & 78 \\
\hline EBUS-GS = endobronchial ultrasonography with guide sheath; \\
GGO = ground-glass opacity.
\end{tabular}

component $>5$ mm was $86.2 \%(25 / 29)$ (Table 4).

\section{Representative case: GGO lesion with a solid com- ponent}

The patient was a 64-year-old man with a highresolution chest CT finding of a $25 \times 23-\mathrm{mm}$ part- solid lesion in the right upper lobe (Fig. 5). The maximum solid-part diameter of the lesion was $5 \mathrm{~mm}$ and the bronchus sign was positive. During EBUS, hyperechoic dots were observed around the probe and scattered throughout the lesion, which was classified as "within." No blood vessels were seen, and the lesion 
Table 4. Solid-part Diameters and EBUS Classifications

\begin{tabular}{|c|c|c|c|c|}
\hline \multirow{2}{*}{$\begin{array}{l}\text { Solid-part } \\
\text { diameter }\end{array}$} & \multicolumn{2}{|c|}{ No. $(\%)$} & \multicolumn{2}{|c|}{ Diagnostic yield (\%) } \\
\hline & Type Ila & Type Ilb & Type Ila & Type IIb \\
\hline $\begin{array}{l}\leq 5 \mathrm{~mm} \\
(\mathrm{n}=10)\end{array}$ & $10(100)$ & $0(0)$ & $6 / 10(60)$ & $0(0)$ \\
\hline $\begin{array}{l}>5 \mathrm{~mm} \\
(\mathrm{n}=43)\end{array}$ & $14(32.6)$ & $29(67.4)$ & $13 / 14(92.9)$ & $25 / 29(86.2)$ \\
\hline
\end{tabular}

EBUS = endobronchial ultrasonography.

The 53 cases classified excluded the 5 invisible cases.

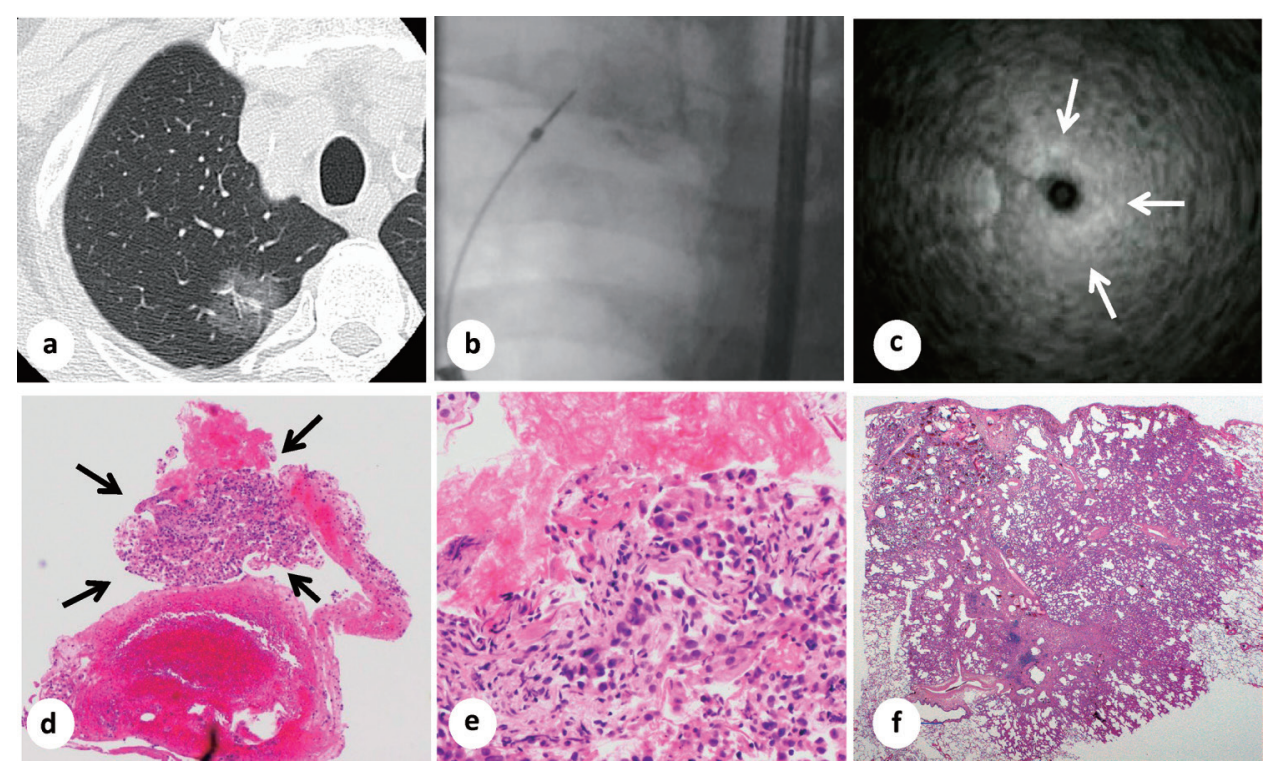

Figure 5. A representative case of EBUS-GS-guided biopsy in a 64-year-old man with a part-solid lesion in the right upper lobe.

a: High resolution CT scan showed a $25-\mathrm{mm}$ part-solid lesion (solid-part diameter $=5 \mathrm{~mm}$ ) in the right upper lobe.

b: Real-time fluoroscopic imaging was performed during EBUS-GS.

c: The EBUS image revealed hyperechoic dots (arrows) around the probe (within). No blood vessels were seen in the Type IIa lesion.

d: The low power histopathologic image of the transbronchial biopsy specimen demonstrated the mass of cells.

e: The high power-histopathologic image of the transbronchial biopsy specimen was consistent with non-small cell carcinoma.

f: The low-power histopathologic image of the surgical specimen showed minimally invasive adenocarcinoma (non-mucinous type). 
was considered a Type IIa lesion. The lesion was confirmed under X-ray fluoroscopic guidance, and brush cytology specimens and biopsy tissue were collected. The patient was diagnosed as having non-small cell carcinoma. Examination of surgical specimens led to a final diagnosis of minimally invasive adenocarcinoma (non-mucinous type).

\section{Discussion}

In this study, we analyzed 58 GGO lesions diagnosed as malignant.The diagnostic yield of EBUSGS-guided biopsy was significantly greater for malignant GGO lesions with a maximum solid-part diameter of $>5 \mathrm{~mm}$ than for lesions with a maximum solid-part diameter of $\leq 5 \mathrm{~mm}$. To the best of our knowledge, no other studies have investigated the specific contribution of CT findings to the diagnosis of GGO lesions achieved simply by means of EBUSGS-guided biopsy. Izumo et al. ${ }^{5)}$ described GGO lesions depicted by EBUS-GS, and Ikezawa et al. ${ }^{4)}$ reported a much greater diagnostic yield for EBUS-GSguided biopsy when lesions were fluoroscopically visible, but these reports pertain strictly to the contribution of EBUS-GS for diagnosis. We investigated the usefulness of CT images to assess the likelihood of an EBUS-GS diagnosis in carefully selected patients with GGO lesions.

We classified the solid part of the lesions by size, $\leq 5 \mathrm{~mm}$ or $>5 \mathrm{~mm}$, for two reasons. First, we referred to Suzuki et al. ${ }^{9)}$, who studied 100 patients with surgically treated adenocarcinoma $\leq 3 \mathrm{~cm}$. They reported a $100 \%$-year survival rate for patients with part-solid lesions having central collapse/fibrosis that were $\leq 5 \mathrm{~mm}$ and a $70 \%$ survival rate for lesions $>5$ $\mathrm{mm}$. The second reason is that Travis et al. ${ }^{10)}$ proposed a new IASLC/ATS/ERS classification for pulmonary adenocarcinoma, and according to that system, they classified a lesion with a solid part $\leq 5 \mathrm{~mm}$ and lesion diameter of $\leq 3 \mathrm{~cm}$ as minimally invasive adenocarcinoma and a lesion with a solid part $>5 \mathrm{~mm}$ as invasive adenocarcinoma or lepidic-predominant adenocarcinoma. The maximum diameter of the solid part was shown to be a significant index. Thus, we used the solid part as an index for this study. In addition, measuring the solid part on CT scans is simply and clinically practical.

The high diagnostic yield for lesions with a solid part $>5 \mathrm{~mm}$ can be explained because most of these lesions are visible on EBUS. First, adenocarcinoma tends to focus around blood vessels and the bronchial invasion into the interstitium ${ }^{11-12)}$. Ikezawa et al. ${ }^{4)}$ studied the relation between lesions and bronchus/ arterial trees observed on high-resolution CT using the classification by Shinagawa et al. ${ }^{13}$. They found the EBUS-GS diagnosis rates to be higher for Type 1 lesions (bronchus leading toward the center of the lesion) than for Types 2-5. Therefore, we hypothesized that bronchi in the lesion with a solid part of $>5 \mathrm{~mm}$ focus more around the tumor and tend to lead inside the lesion, enhancing the visibility on EBUS images and facilitating diagnosis.

Second, lesions with a solid component $>5 \mathrm{~mm}$ were mostly Type IIb lesions (Table 4). When welldifferentiated adenocarcinoma replaces the alveolar lining, air remains in the alveolus, and this is recognized as hyperechoic dots on the image; therefore, we can classify the lesion as Type II. We believe air in the alveolus may disturb the visualization of blood vessels in Type IIa lesions, hindering diagnosis. In Type IIb lesions, the density of tumor cells is high, the volume of air in the alveolus is decreased, and tumor cells can multiply while blood vessels are maintained $^{8}$. We believe that the density of tumor cells, and the degree of cell heteromorphy is greater in Type IIb adenocarcinoma, which increases the diagnostic yield. Suzuki et al. ${ }^{9)}$ confirmed that a solid component $>5 \mathrm{~mm}$ was predictive of an adverse outcome. Type IIb includes interstitial infiltration of tumor cells, and therefore, Type IIb may be useful as a prognostic factor.

There were lesions with a solid part $>5 \mathrm{~mm}$ that were invisible on EBUS. We think that the probe was inserted into the incorrect bronchus or that the GGO was too faint to produce hyperechoic dots on EBUS. There were also lesions for which EBUS images were obtained but there was no resulting diagnosis. This was probably because the biopsy specimen was actually of the bronchus wall and not of the lesion.

CT-guided biopsy is widely used in Europe and the United States for the diagnosis of PPLs. Shimizu et al. ${ }^{14)}$ investigated the diagnosis of GGO lesions based on CT-guided biopsy and reported a diagnostic rate of $51.2 \%$ in the "GGO-dominant" group and $75.6 \%$ in the "solid-dominant" group. They reported a complication rate of $34.4 \%$, with $12.5 \%$ of patients requiring placement of a chest drain and $3.1 \%$ suffering pulmonary hemorrhage ${ }^{14}$. Our study results were similar to the reported results of CT-guided biopsy, but without patient complications. EBUS-GS-guided biopsy has several advantages over transbronchial biopsy and CT-guided biopsy. First, EBUS-GS-guided biopsy is readily repeatable to access to the bronchial 
lesion for sampling. Second, the advantage of EBUSGS-guided biopsy is in its ability to protect against bleeding into the proximal bronchus from the biopsy site. Further, the risks associated with EBUS-GS-guided biopsy are very low when compared with CTguided biopsy, and there is no radiation exposure.

Our study was subject to the potential biases of any retrospective study. To further investigate our study questions and confirm our findings, we will need to conduct a prospective study with a larger patient group. Furthermore, additional research questions should be pursued, such as whether differences in GGO shading on CT images affect diagnostic rates of EBUS-GS, and whether alveolar collapse and interstitial fibrosis can be differentiated during the progression of lung cancer. Analysis of radio-frequency signals of original data from lesions and histograms of lesion brightness may also be useful for such differentiation on EBUS images.

\section{Conclusions}

We showed that the diagnostic yield is significantly greater when the maximum diameter of the solid component of a malignant GGO lesion on a CT image is $>5 \mathrm{~mm}$. The information obtained from CT can improve the potential for an EBUS-GS-guided biopsy diagnosis of pulmonary malignant GGO lesions.

\section{Acknowledgments}

The authors thank Tina Tajima of St. Marianna University School of Medicine for her editorial review of the English manuscript. The authors also thank Shinobu Tatsunami of the Medical Statistics Department at St. Marianna University School of Medicine for her valuable advice on the statistical analysis.

\section{Financial Disclosure and Conflicts of Interest}

All authors have no conflicts of interest to declare.

\section{References}

1) National Lung Screening Trial Research Team, Aberle DR, Adams AM, Berg CD, Black WC, Clapp JD, Fagerstrom RM, Gareen IF, Gatsonis C, Marcus PM, Sicks JD. Reduced lung-cancer mortality with low-dose computed tomographic screening. N Engl J Med 2011; 4: 395-409.

2) Wahidi MM, Govert JA, Goudar RK, Gould MK, McCrory DC; American College of Chest
Physicians. Evidence for the treatment of patients with pulmonary nodules: when is it lung cancer?: ACCP evidence-based clinical practice guidelines. CHEST 2007; 132 (3suppl): 94S$107 \mathrm{~S}$.

3) Kurimoto $N$, Miyazawa $T$, Okimasa $S$, Maeda A, Oiwa H, Miyazu Y, Murayama M. Endobronchial ultrasonography using a guide sheath increases the ability to diagnose peripheral pulmonary lesions endoscopically. CHEST 2004; 126: 959-965.

4) Ikezawa $Y$, Sukoh N, Shinagawa N, Nakano K, Oizumi S, Nishimura M. Endobronchial ultrasonography with a guide sheath for pure or mixed ground-glass opacity lesions. Respiration 2014; 88: 137-143.

5) Izumo $T$, Sasada $S$, Chavez $C$, Tsuchida $T$. The diagnostic utility of endobronchial ultrasonography with a guide sheath and tomosynthesis images for ground glass opacity pulmonary lesions. J Thorac Dis 2013; 5: 745-750.

6) Naidich DP, Bankier AA, MacMahon H, Schaefer-Prokop CM, Pistolesi M, Goo JM, Macchiarini P, Crapo JD, Herold CJ, Austin JH. Recommendations for the management of subsolid pulmonary nodules detected at CT: A statement from the fleischner society. Radiology 2013; 266: 304-317.

7) Naidich DP, Sussman R, Kutcher WL, Aranda CP, Garay SM, Ettenger NA. Solitary pulmonary nodules: CT-bronchoscopic correlation. CHEST 1988; 93: 595-598.

8) Kurimoto N, Murayama M, Yoshioka S, Nishisaka T. Analysis of the internal structure of peripheral pulmonary lesions using endobronchial ultrasonography. CHEST 2002; 122: $1887-$ 1894.

9) Suzuki K, Yokose T, Yoshida J, Nishimura M, Takahashi K, Nagai K, Nishiwaki Y. Prognostic significance of the size of central fibrosis in peripheral adenocarcinoma of the lung. Ann Thorac Surg 2000; 69: 893-897.

10) Travis WD, Brambilla E, Noguchi M, Nicholson AG, Geisinger K, Yatabe Y, Powell CA, Beer D, Riely G, Garg K. International association for the study of lung Cancer/American thoracic Society/European respiratory society: International multidisciplinary classification of lung adenocarcinoma: Executive summary. Proceedings of American Thoracic Society 2011; 8: 381-385.

11) Kitaoka H, Takaki R. Simulations of bronchial 
displacement owing to solitary pulmonary nodules. Jpn J Radiol 1999; 59: 318-324.

12) Matsumoto T, Tajima $Y$, Eguchi T, Hirata M. Clinicopathological studies on subtypes of adenocarcinoma of the lung and 'corrected tumor size'. JJLC 1980; 20: 163-169.

13) Shinagawa N, Yamazaki K, Onodera Y, Asahina H, Kikuchi E, Asano F, Miyasaka K, Nishimura
M. Factors related to diagnostic sensitivity using an ultrathin bronchoscope under CT guidance. CHEST 2007; 131: 549-553.

14) Shimizu K, Ikeda N, Tsuboi M, Hirano T, Kato H. Percutaneous CT-guided fine needle aspiration for lung cancer smaller than $2 \mathrm{~cm}$ and revealed by ground-glass opacity at CT. Lung Cancer 2006; 51: 173-179. 